\title{
A Bending-Gradient theory for thick laminated plates homogenization
}

\author{
A. Lebée, K. Sab
}

\begin{abstract}
This work presents a new plate theory for out-of-plane loaded thick plates where the static unknowns are those of the Love-Kirchhoff theory, to which six components are added representing the gradient of the bending moment. The BendingGradient theory is an extension to arbitrary multilayered plates of the ReissnerMindlin theory which appears as a special case when the plate is homogeneous. The new theory is applied to multilayered plates and its predictions are compared to full 3D Pagano's exact solutions and other approaches. It gives good predictions of both deflection and shear stress distributions in any material configuration. Moreover, under some symmetry conditions, the Bending-Gradient model coincides with the second-order approximation of the exact solution as the slenderness ratio $L / h$ goes to infinity.
\end{abstract}

\section{Introduction}

Laminated plates are widely used in engineering applications. For instance angleply carbon fiber reinforced laminates are commonly used in aeronautics. However, these materials are strongly anisotropic and the plate overall behavior is difficult to capture. The most common plate theory is the Love-Kirchhoff plate model. However, it is well-known that, when the plate slenderness ratio $L / h$ is not large enough, transverse shear stresses which are not taken into account in the Love-Kirchhoff theory have an increasing influence on the plate deflection.

\footnotetext{
A. Lebée

Université Paris-Est, Laboratoire Navier (ENPC/LCPC/CNRS). École des Ponts ParisTech, 6 et 8 avenue Blaise Pascal. 77455 Marne-la-Vallée, e-mail:arthur.lebee@enpc.fr

K. Sab

Université Paris-Est, Laboratoire Navier (ENPC/LCPC/CNRS). École des Ponts ParisTech, 6 et 8 avenue Blaise Pascal. 77455 Marne-la-Vallée, e-mail: karam.sablenpc.fr
} 
In recent decades many suggestions have been made to improve the estimation of transverse shear stresses. Two main approaches can be found: asymptotic approaches and axiomatic approaches. The first one is mainly based on asymptotic expansions in the small parameter $h / L$ [2, 3]. However, no distinction between relevant fields and unknowns was made. The second main approach is based on assuming ad hoc displacement or stress 3D fields. These models can be "Equivalent Single Layer" or "Layerwise". Equivalent single layer models treat the whole laminate as an equivalent homogeneous plate. However, when dealing with laminated plates, these models lead systematically to discontinuous transverse shear stress distributions through the thickness as indicated by Reddy [4]. In Layerwise models, all plate degrees of freedom are introduced in each layer of the laminate and continuity conditions are enforced between layers. The reader can refer to Reddy [4] and Carrera [5] for detailed reviews of kinematic approaches and to [6, 7, 8] for static approaches. Layerwise models lead to correct estimates of local 3D fields. However, their main drawback is that they involve a number of degrees of freedom proportional to the number of layers. The limitation is immediately pointed out with functionally graded materials, where the plate constituents properties vary continuously through the thickness [9, 10].

Based on Reissner [11] paper, we suggest an Equivalent Single Layer higherorder plate theory which gives an accurate enough estimate of transverse shear stresses in the linear elasticity framework. For this, we are motivated by two observations. The first one is that Love-Kirchhoff strain fields have clearly been identified as good first-order approximation for slender plates thanks to asymptotic expansion approaches. The second one is that the $3 \mathrm{D}$ equilibrium plays a critical role in the estimation of transverse shear stress in all the existing approaches. We show in this work that revisiting the use of 3D equilibrium in order to derive transverse shear stress as Reissner [11] did for homogeneous plates leads to a full bending gradient plate theory. The Reissner-Mindlin theory is as a special case of the new BendingGradient theory when the plate is homogeneous.

In Section 2 notations are introduced. In Section 3] we resume Reissner]'s procedure for deriving transverse shear stress extended to laminated plates. This lead to the Bending-Gradient plate theory detailed in Section 4 Finally, in Section 5 the Bending-Gradient plate theory is applied to fibrous laminates under cylindrical bending and compared to the exact solution and other Single Equivalent Layer approaches.

\section{Notations}

Vectors and higher-order tensors are boldfaced and different typefaces are used for each order: vectors are slanted: $\boldsymbol{T}, \boldsymbol{u}$. Second order tensors are sans serif: M, e. Third order tensors are in typewriter style: $\boldsymbol{\Phi}, \Gamma$. Fourth order tensors are in calligraphic style $\mathcal{D}, \boldsymbol{c}$. Sixth order tensors are double stroked $\mathbb{F}, \mathbb{W}$. 
When dealing with plates, both 2-dimensional (2D) and 3D tensors are used. Thus, $\widetilde{\boldsymbol{T}}$ denotes a $3 \mathrm{D}$ vector and $\boldsymbol{T}$ denotes a $2 \mathrm{D}$ vector or the in-plane part of $\widetilde{\boldsymbol{T}}$. The same notation is used for higher-order tensors: $\widetilde{\boldsymbol{\sigma}}$ is the 3D second-order stress tensor while $\boldsymbol{\sigma}$ is its in-plane part. When dealing with tensor components, the indexes specify the dimension: $\mathrm{a}_{i j}$ denotes the $3 \mathrm{D}$ tensor $\widetilde{\mathbf{a}}$ with Latin index $i, j, k . .=1,2,3$ and $\mathrm{a}_{\alpha \beta}$ denotes the $2 \mathrm{D}$ a tensor with Greek indexes $\alpha, \beta, \gamma_{.}=1,2 . \widetilde{\boldsymbol{C}}=C_{i j k l}$ is the fourth-order 3D elasticity stiffness tensor. $\widetilde{\mathcal{S}}=S_{i j k l}=\widetilde{\mathcal{C}}^{-1}$ is the fourth-order 3D elasticity compliance tensor while $c=c_{\alpha \beta \gamma \delta}$ denotes the plane-stress elasticity tensor. $c$ is not the in-plane part of $\widetilde{\boldsymbol{C}}$ but it is the inverse of the in-plane part of $\widetilde{\mathcal{S}}$ : $\boldsymbol{c}=\mathcal{S}^{-1}$. The identity for in-plane elasticity is $i_{\alpha \beta \gamma \delta}=\frac{1}{2}\left(\delta_{\alpha \gamma} \delta_{\beta \delta}+\delta_{\alpha \delta} \delta_{\beta \gamma}\right)$, where $\delta_{\alpha \beta}$ is Kronecker symbol ( $\delta_{\alpha \beta}=1$ if $\alpha=\beta, \delta_{\alpha \beta}=0$ otherwise).

The transpose operation ${ }^{t} \bullet$ is applied to any order tensors as follows: $\left({ }^{t} A\right)_{\alpha \beta \ldots \psi \omega}=$ $A_{\omega \psi \ldots \beta \alpha}$.

Three contraction products are defined, the usual dot product $\left(\widetilde{\boldsymbol{a}} \cdot \widetilde{\boldsymbol{b}}=a_{i} b_{i}\right)$, the double contraction product $\left(\widetilde{\mathbf{a}}: \widetilde{\mathbf{b}}=\mathrm{a}_{i j} \mathrm{~b}_{j i}\right)$ and a triple contraction product $(\mathbf{A} \therefore \mathbf{B}=$ $\mathrm{A}_{\alpha \beta \gamma} \mathrm{B}_{\gamma \beta \alpha}$ ). In these definitions Einstein's notation on repeated indexes is used. It should be noticed that closest indexes are summed together in contraction products. Thus, $\boldsymbol{\Phi} \cdot \boldsymbol{n}=\Phi_{\alpha \beta \gamma} n_{\gamma}$ is different from $\boldsymbol{n} \cdot \boldsymbol{\Phi}=n_{\alpha} \Phi_{\alpha \beta \gamma}$. The derivation operator $\widetilde{\boldsymbol{\nabla}}$ is also formally represented as a vector: $\widetilde{\mathbf{a}} \cdot \widetilde{\boldsymbol{\nabla}}=\mathrm{a}_{i j} \nabla_{j}=\mathrm{a}_{i j, j}$ is the divergence and $\widetilde{\mathbf{a}} \otimes \widetilde{\boldsymbol{\nabla}}=\mathrm{a}_{i j} \nabla_{k}=\mathrm{a}_{i j, k}$ is the gradient. Here $\otimes$ is the dyadic product. Finally, the integration through the thickness is noted $\langle\bullet\rangle: \int_{-\frac{h}{2}}^{\frac{h}{2}} f\left(x_{3}\right) d x_{3}=\langle f\rangle$.

\section{Revisiting the Reissner-Mindlin plate theory}

\section{The 3D model}

We consider a linear elastic plate of thickness $h$ occupying the 3D domain $\Omega=$ $\omega \times]-h / 2, h / 2$, where $\omega \subset \mathbb{R}^{2}$ is the mid-plane of the plate (Figure11. Cartesian coordinates $\left(x_{1}, x_{2}, x_{3}\right)$ in the reference frame $\left(\widetilde{\boldsymbol{e}}_{1}, \widetilde{\boldsymbol{e}}_{2}, \widetilde{\boldsymbol{e}}_{3}\right)$ are used. The constitutive material is assumed to be invariant with respect to translations in the $\left(x_{1}, x_{2}\right)$ plane. Hence, the stiffness tensor $\widetilde{C}$ is a function of $x_{3}$ only. The plate is loaded on its upper and lower faces $\omega^{ \pm}=\omega \times\{ \pm h / 2\}$ with the distributed force $\widetilde{\boldsymbol{T}}^{ \pm}$. There are no body forces and the plate is clamped on its lateral edge, $\partial \omega \times]-h / 2, h / 2[$ where $\partial \omega$ is the edge of $\omega$. The $3 \mathrm{D}$ problem $\mathscr{P}^{3 D}$ is summarized as follows: 


$$
\mathscr{P}^{3 D}\left\{\begin{array}{l}
\tilde{\boldsymbol{\sigma}} \cdot \widetilde{\boldsymbol{\nabla}}=0 \quad \text { on } \Omega . \\
\widetilde{\boldsymbol{\sigma}}=\widetilde{\boldsymbol{C}}\left(x_{3}\right): \widetilde{\boldsymbol{\varepsilon}} \quad \text { on } \Omega . \\
\widetilde{\boldsymbol{\sigma}} \cdot \widetilde{\boldsymbol{e}}_{3}=\widetilde{\boldsymbol{T}}^{ \pm} \quad \text { on } \omega^{ \pm} . \\
\widetilde{\boldsymbol{\varepsilon}}=\frac{1}{2}(\widetilde{\boldsymbol{\nabla}} \otimes \widetilde{\boldsymbol{u}}+\widetilde{\boldsymbol{u}} \otimes \widetilde{\nabla}) \quad \text { on } \Omega . \\
\widetilde{\boldsymbol{u}}=0 \quad \text { on } \partial \omega \times]-h / 2, h / 2[.
\end{array}\right.
$$

where $\tilde{\boldsymbol{u}}$ is the 3D displacement vector field, $\widetilde{\boldsymbol{\varepsilon}}$ is the strain tensor field and $\widetilde{\boldsymbol{\sigma}}$ is the stress tensor field.

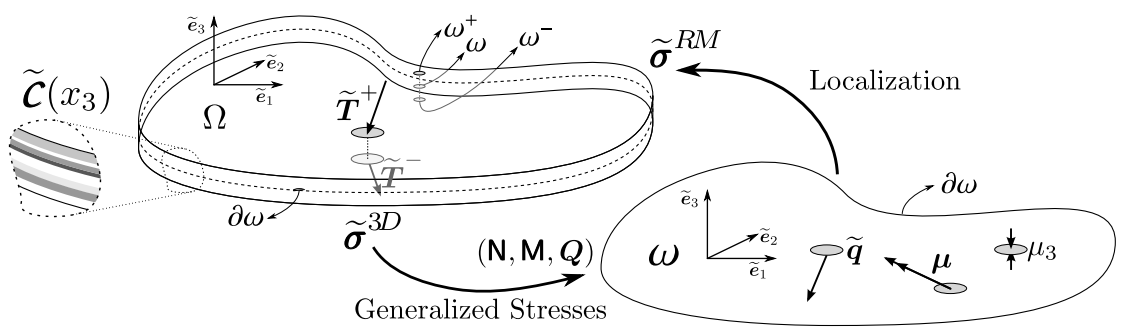

Fig. 1 The Plate Configuration

Reissner-Mindlin statically compatible fields

We recall here briefly the procedure for the derivation of Reissner-Mindlin equilibrium equations [11, 12, 13]. The generalized Reissner-Mindlin stresses associated to the 3D stress field $\widetilde{\boldsymbol{\sigma}}$ are:

$$
\begin{gathered}
\mathrm{N}_{\alpha \beta}\left(x_{1}, x_{2}\right)=\left\langle\sigma_{\alpha \beta}\right\rangle \\
\mathrm{M}_{\alpha \beta}\left(x_{1}, x_{2}\right)=\left\langle x_{3} \sigma_{\alpha \beta}\right\rangle \\
Q_{\alpha}\left(x_{1}, x_{2}\right)=\left\langle\sigma_{\alpha 3}\right\rangle
\end{gathered}
$$

where $\mathbf{N}$ is the membrane stress, $\mathbf{M}$ the bending moment, and $\boldsymbol{Q}$ the shear forces.

Reissner-Mindlin equilibrium equations are obtained by integrating equations 1a and $x_{3} \times 1$ with respect to $x_{3}$. Taking also into account boundary conditions 1c yields:

$$
\left\{\begin{array}{l}
\mathrm{N}_{\alpha \beta, \beta}+q_{\alpha}=0 \\
Q_{\alpha, \alpha}+q_{3}=0 \\
\mathrm{M}_{\alpha \beta, \beta}-Q_{\alpha}+\mu_{\alpha}=0
\end{array}\right.
$$


where $q_{i}=T_{i}^{+}+T_{i}^{-}$are symmetric loadings per unit surface and $\mu_{i}=\frac{h}{2}\left(T_{i}^{+}-\right.$ $\left.T_{i}^{-}\right)$are skew-symmetric loadings per unit surface. More precisely, $\boldsymbol{q}=\left(q_{\alpha}\right)$ are membrane loadings per unit surface, $q_{3}$ is the out-of-plane loading per unit surface, $\boldsymbol{\mu}=\left(\mu_{\alpha}\right)$ are couples per unit surface and $\mu_{3}$ is the transverse bulk loading.

Since in-plane loadings $(\boldsymbol{q}, \boldsymbol{\mu})$ and out-of-plane loadings $\left(q_{3}, \mu_{3}\right)$ are not of the same order in the asymptotic analysis of the plate as $h / L$ goes to 0 (see [3]), and for the sake of simplicity, we focus only on the out-of-plane loading $q_{3}\left(q_{\alpha}=\mu_{i}=0\right)$.

\section{Localization}

The second step of Reissner's approach consists in deriving the stress energy per unit surface $w^{* R M}(\mathbf{N}, \mathbf{M}, \boldsymbol{Q})$ from the 3D model. As in many homogenization procedures, the derivation of $w^{* R M}$ is based on an approximation scheme for the real 3D stress fields in terms of the generalized plate stress fields:

$$
\tilde{\boldsymbol{\sigma}}^{R M}\left(x_{1}, x_{2}, x_{3}\right)=\tilde{\boldsymbol{\sigma}}^{(\mathrm{N})}\left(x_{1}, x_{2}, x_{3}\right)+\tilde{\boldsymbol{\sigma}}^{(\mathrm{M})}\left(x_{1}, x_{2}, x_{3}\right)+\tilde{\boldsymbol{\sigma}}^{(Q)}\left(x_{1}, x_{2}, x_{3}\right)
$$

where $\tilde{\boldsymbol{\sigma}}^{(\mathrm{N})}, \widetilde{\boldsymbol{\sigma}}^{(\mathrm{M})}$, and $\tilde{\boldsymbol{\sigma}}^{(Q)}$ are 3D stress fields generated by $\mathrm{N}, \mathbf{M}$ and $\boldsymbol{Q}$ as follows:

$$
\left\{\begin{array}{l}
\sigma_{i j}^{(\mathrm{N})}=s_{i j \alpha \beta}^{(\mathrm{N})}\left(x_{3}\right) \mathrm{N}_{\alpha \beta}\left(x_{1}, x_{2}\right) \\
\sigma_{i j}^{(\mathrm{M})}=s_{i j \alpha \beta}^{(\mathrm{M})}\left(x_{3}\right) \mathrm{M}_{\alpha \beta}\left(x_{1}, x_{2}\right) \\
\sigma_{i j}^{(Q)}=s_{i j \alpha}^{(Q)}\left(x_{3}\right) Q_{\alpha}\left(x_{1}, x_{2}\right)
\end{array}\right.
$$

where $s_{i j \alpha \beta}^{(\mathrm{N})}\left(x_{3}\right), s_{i j \alpha \beta}^{(\mathrm{M})}\left(x_{3}\right)$ and $s_{i j \alpha}^{(Q)}\left(x_{3}\right)$ are localization tensors depending only on the $x_{3}$ coordinate. This can be rewritten using contraction products as:

$$
\widetilde{\boldsymbol{\sigma}}^{R M}=\widetilde{\boldsymbol{s}}^{(\mathrm{N})}: \mathbf{N}+\widetilde{\boldsymbol{s}}^{(\mathrm{M})}: \mathbf{M}+\widetilde{\boldsymbol{s}}^{(Q)} \cdot \boldsymbol{Q}
$$

Once this approximation of stress fields is set, the stress potential energy density $w^{* R M}(\mathbf{N}, \mathbf{M}, \boldsymbol{Q})$ is defined simply as the quadratic form:

$$
w^{* R M}(\mathbf{N}(\boldsymbol{x}), \mathbf{M}(\boldsymbol{x}), \boldsymbol{Q}(\boldsymbol{x}))=\frac{1}{2}\left\langle\tilde{\boldsymbol{\sigma}}^{R M}(\widetilde{\boldsymbol{x}}): \tilde{\boldsymbol{S}}\left(x_{3}\right): \tilde{\boldsymbol{\sigma}}^{R M}(\widetilde{\boldsymbol{x}})\right\rangle
$$

Hence, a consistent choice for $\widetilde{\boldsymbol{s}}^{(\mathrm{N})}, \widetilde{\boldsymbol{s}}^{(\mathrm{M})}$ and $\widetilde{\boldsymbol{s}}^{(Q)}$ is critical.

\section{Love-Kirchhoff fields}

The derivation of $\widetilde{\boldsymbol{s}}^{(\mathrm{N})}$ and $\widetilde{\boldsymbol{s}}^{(\mathrm{M})}$ is based on the Love-Kirchhoff plate theory. According to this theory, plane-stress is assumed and the in-plane part of the strain is linear in $x_{3}$ : 


$$
\boldsymbol{\varepsilon}^{L K}=\mathbf{e}+x_{3} \boldsymbol{\chi}
$$

where $\mathbf{e}$ is the membrane strain and $\chi$ the curvature. We draw the reader's attention to the fact that strain components $\varepsilon_{i 3}$ are not null in the general case. Membrane stress $\mathbf{N}$ and bending moments $\mathbf{M}$ are linearly dependent on $\mathbf{e}$ and $\boldsymbol{\chi}$ :

$$
\left\{\begin{array}{l}
\mathrm{N}=\mathcal{A}: \mathrm{e}+\mathcal{B}: \boldsymbol{\chi} \\
\mathrm{M}={ }^{t} \mathcal{B}: \mathrm{e}+\mathcal{D}: \boldsymbol{\chi}
\end{array}\right.
$$

with:

$$
(\mathcal{A}, \mathcal{B}, \mathcal{D})=\left\langle\left(1, x_{3}, x_{3}^{2}\right) \boldsymbol{c}\left(x_{3}\right)\right\rangle
$$

Using 3D constitutive equation under plane-stress assumption, Love-Kirchhoff constitutive equation (9) and in-plane strains definition (8), it is possible to express Love-Kirchhoff stress fields as functions of $\mathbf{N}$ and $\mathbf{M}$ :

$$
\begin{cases}\boldsymbol{\sigma}^{(\mathrm{N})}\left(x_{1}, x_{2}, x_{3}\right)=\boldsymbol{c}\left(x_{3}\right):\left(\boldsymbol{a}+x_{3}{ }^{t} \boldsymbol{b}\right): \mathbf{N}\left(x_{1}, x_{2}\right) & \text { and } \sigma_{i 3}^{(\mathrm{N})}=0 \\ \boldsymbol{\sigma}^{(\mathrm{M})}\left(x_{1}, x_{2}, x_{3}\right)=\boldsymbol{c}\left(x_{3}\right):\left(\boldsymbol{b}+x_{3} \boldsymbol{d}\right): \mathbf{M}\left(x_{1}, x_{2}\right) & \text { and } \sigma_{i 3}^{(\mathrm{M})}=0\end{cases}
$$

where $\boldsymbol{a}, \boldsymbol{b}$ and $\boldsymbol{d}$ are the reciprocal compliance tensors of the constitutive equation (9).

Stress field generated by a linear variation of the bending moment

The main idea of Reissner's method is to recall that the shear forces are related to the bending moment through the plate equilibrium (5). With a homogeneous plate, combining both $3 \mathrm{D}$ equilibrium and plate equilibrium enables the derivation of a stress field directly depending on shear forces. However, with laminated plates it is not possible to bring out shear force with this procedure. Here, we suggest considering a more general shear variable for laminates, the full bending gradient: $\mathbf{R}=\mathbf{M} \otimes \boldsymbol{\nabla}$. In the following, we resume the procedure from Reissner [11] for deriving shear fields in the case of laminated plates.

We have $\tilde{\boldsymbol{\sigma}}^{(\mathrm{M})} \cdot \widetilde{\boldsymbol{\nabla}}=0$ if $\mathbf{M}$ is $\left(x_{1}, x_{2}\right)$-invariant. When $\mathbf{M}$ is function of $x_{1}$ and $x_{2}$, we have:

$$
\tilde{\boldsymbol{\sigma}}^{(\mathrm{M})} \cdot \widetilde{\nabla}=s_{i j \beta \alpha}^{(\mathrm{M})}\left(x_{3}\right) \mathrm{M}_{\alpha \beta}\left(x_{1}, x_{2}\right) \nabla_{j}=s_{i j \beta \alpha}^{(\mathrm{M})} \mathrm{M}_{\alpha \beta, \gamma} \delta_{j \gamma}=s_{i \gamma \beta \alpha}^{(\mathrm{M})} \mathrm{R}_{\alpha \beta \gamma}
$$

$f_{i}^{(\mathrm{R})}=s_{i \gamma \beta \alpha}^{(\mathrm{M})} \mathrm{R}_{\alpha \beta \gamma}$ is the force per unit volume generated by first order variations of the bending moment $R$. $R_{\alpha \beta \gamma}$ is a third-order tensor which respects $M_{\alpha \beta}$ symmetries $\left(\mathrm{R}_{\alpha \beta \gamma}=\mathrm{R}_{\beta \alpha \gamma}\right)$. Using $\tilde{\boldsymbol{\sigma}}^{(\mathrm{M})}$ definition (Equation $11 \mathrm{~b}$ ) and assuming that each layer follows monoclinic symmetry we identify the force per unit volume as:

$$
\boldsymbol{f}^{(\mathrm{R})}=\boldsymbol{c}\left(x_{3}\right):\left(\boldsymbol{b}+x_{3} \boldsymbol{d}\right) \therefore \mathbf{R} \text { and } f_{3}^{(\mathrm{R})}=0
$$


Then, we define $\tilde{\boldsymbol{\sigma}}^{(\mathrm{R})}$ the 3D stress generated by a $\left(x_{1}, x_{2}\right)$-invariant bending gradient R associated to the localization tensor $s_{i j \alpha \beta \gamma}^{(\mathrm{R})}$ such as $\widetilde{\boldsymbol{\sigma}}^{(\mathrm{R})}=\widetilde{\boldsymbol{s}}^{(\mathrm{R})} \therefore \mathrm{R}$. This stress field is derived through the auxiliary problem:

$$
\left\{\begin{array}{l}
\tilde{\boldsymbol{\sigma}}^{(\mathrm{R})} \cdot \widetilde{\boldsymbol{\nabla}}+\widetilde{\boldsymbol{f}}^{(\mathrm{R})}=\widetilde{\mathbf{0}} \\
\widetilde{\boldsymbol{\sigma}}^{(\mathrm{R})} \cdot \widetilde{\boldsymbol{e}}_{3}=\widetilde{\mathbf{0}} \text { for } x_{3}= \pm h / 2
\end{array}\right.
$$

The $\left(x_{1}, x_{2}\right)$-invariant solution of this problem is easily found, leading to the explicit determination of $\widetilde{\boldsymbol{s}}^{(\mathrm{R})}$ :

$$
s_{\alpha 3 \eta \zeta \varepsilon}^{(\mathrm{R})}\left(x_{3}\right)=-\int_{-\frac{h}{2}}^{x_{3}} c_{\alpha \eta \gamma \delta}(z)\left(b_{\delta \gamma \varepsilon \zeta}+z d_{\delta \gamma \varepsilon \zeta}\right) d z, s_{\alpha \beta \eta \zeta \varepsilon}^{(\mathrm{R})}=0 \text { and } s_{33 \eta \zeta \varepsilon}^{(\mathrm{R})}=0
$$

We have derived a localization tensor $\widetilde{\boldsymbol{s}}^{(\mathrm{R})}$ which depends on all bending gradient components: $\mathrm{R}_{\alpha \beta \gamma}=\mathrm{M}_{\alpha \beta, \gamma}$. Accordingly we define a new approximation of stress fields involving all bending gradient components:

$$
\tilde{\boldsymbol{\sigma}}^{B G}=\tilde{\boldsymbol{\sigma}}^{(\mathrm{N})}+\tilde{\boldsymbol{\sigma}}^{(\mathrm{M})}+\tilde{\boldsymbol{\sigma}}^{(\mathrm{R})}
$$

and a new stress energy density identical to Definition 7 .

$$
w^{* B G}(\mathbf{N}, \mathbf{M}, \mathbf{R})
$$

Actually $\widetilde{\boldsymbol{\sigma}}^{B G}$ approximation for 3D stress fields is a higher-order gradient theory, as described in [14] for 3D continuum and [15] for periodic beams. However, to be consistent with higher-order theories, we should have taken into account the gradient of other static unknowns such as the membrane stress gradient for instance. It is the choice of the authors to limit the number of static variables only to those which have a contribution to the macroscopic equilibrium of the plate. Thus the number of unknowns remains limited and adapted to engineering applications, contrary to asymptotic expansions and other rigorous approaches in which no distinction is made between significant static unknowns.

The mechanical meaning of the gradient of the bending moment

The full bending gradient $R$ has six components $\left(R_{111}, R_{221}, R_{121}, R_{112}, R_{222}, R_{122}\right)$ whereas $\boldsymbol{Q}$ has two components. Thus, using the full bending gradient as static unknown introduces four static unknowns which a priori are not related to plate equilibrium 5c. Only $(\mathbf{N}, \mathbf{M}, \boldsymbol{Q})$ appeared while integrating 3D equilibrium equation (1a) through the thickness in Section 3

Let us derive generalized stresses associated to $\tilde{\boldsymbol{\sigma}}^{(\mathrm{R})}$. Using Equation 14 and integrating by parts when necessary leads to:

$$
\left\langle\sigma_{\alpha \beta}^{(\mathrm{R})}\right\rangle=0, \quad\left\langle x_{3} \sigma_{\alpha \beta}^{(\mathrm{R})}\right\rangle=0, \quad\left\langle s_{\alpha 3 \beta \gamma \delta}^{(\mathrm{R})}\right\rangle=i_{\alpha \beta \gamma \delta}
$$


and we have: $\left\langle\sigma_{\alpha 3}^{(\mathrm{R})}\right\rangle=\boldsymbol{i} \therefore \mathrm{R}=\boldsymbol{Q}$. Only $\boldsymbol{Q}$ remains after integrating $\tilde{\boldsymbol{\sigma}}^{(\mathrm{R})}$ through the thickness and the four other static unknowns are self-equilibrated stress. More precisely we have:

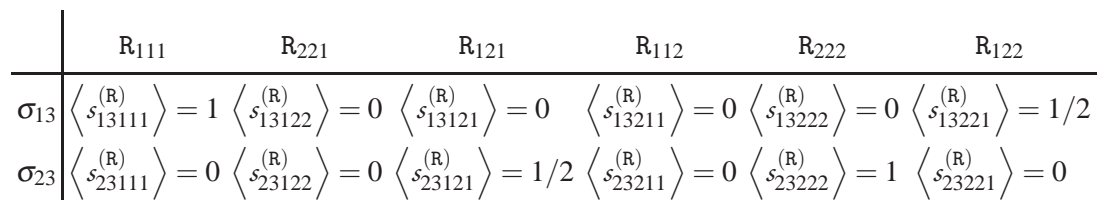

$\mathrm{R}_{111}$ and $\mathrm{R}_{222}$ are the cylindrical bending part of shear forces $Q_{1}$ and $Q_{2}, \mathrm{R}_{121}$ and $R_{122}$ are the torsion part of shear forces and $R_{112}$ and $R_{221}$ are linked to strictly self-equilibrated stresses (warping).

\section{The Bending-Gradient plate model}

Once stress energy density $w^{* B G}(\mathbf{N}, \mathbf{M}, \mathbf{R})$ and plate equilibrium equations 5 to which is added $\mathbf{R}=\mathbf{M} \otimes \nabla$ are given it is possible to build a complete plate theory using conventional variational tools. The reader is refered to [16] for details.

Summary of the plate model

Equilibrium equations and boundary conditions involving stress fields are gathered in the set of statically compatible fields:

$$
\left\{\begin{array}{l}
\mathbf{N} \cdot \boldsymbol{\nabla}=\mathbf{0} \text { on } \omega \\
\mathbf{M} \otimes \boldsymbol{\nabla}-\mathbf{R}=0 \text { on } \omega \\
(\boldsymbol{i} \therefore \mathbf{R}) \cdot \boldsymbol{\nabla}=-q_{3} \text { on } \omega \\
\mathbf{N} \cdot \boldsymbol{n}=\boldsymbol{V}^{d} \text { on } \partial \omega^{s} \\
\mathbf{M}=\mathbf{M}^{d} \text { on } \partial \omega^{s} \\
(\boldsymbol{i} \therefore \mathbf{R}) \cdot \boldsymbol{n}=V_{3}^{d} \text { on } \partial \omega^{s}
\end{array}\right.
$$

where $\partial \omega^{s}$ is the portion of edge on which static boundary conditions apply: $\widetilde{\boldsymbol{V}}^{d}$ is the force per unit length and $\mathbf{M}^{d}$ the full bending moment enforced on the edge. This set of equations is almost identical to Reissner-Mindlin equations where shear forces have been replaced by the bending gradient $\mathbf{R}$.

Generalized stresses $\mathbf{N}, \mathbf{M}$, and $\mathbf{R}$ work respectively with the associated strain variables: $\mathrm{e}$, the conventional membrane strain, $\chi$ the conventional curvature and $\Gamma$ the generalized shear strain. These strain fields must comply with the following 
compatibility conditions and boundary conditions:

$$
\left\{\begin{array}{l}
\mathbf{e}=\boldsymbol{i}:(\boldsymbol{\nabla} \otimes \boldsymbol{U}) \text { on } \omega \\
\boldsymbol{\chi}=\boldsymbol{\Phi} \cdot \boldsymbol{\nabla} \text { on } \omega \\
\boldsymbol{\Gamma}=\boldsymbol{\Phi}+\boldsymbol{i} \cdot \boldsymbol{\nabla} U_{3} \text { on } \omega \\
\boldsymbol{\Phi} \cdot \boldsymbol{n}=\mathbf{H}^{d} \text { on } \partial \omega^{k} \\
\widetilde{\boldsymbol{U}}=\widetilde{\boldsymbol{U}}^{d} \text { on } \partial \omega^{k}
\end{array}\right.
$$

where $\widetilde{\boldsymbol{U}}$ is the 3D displacement of the mid-plane of the plate and $\Phi$ is the generalized rotation. $\Gamma$ and $\Phi$ are 2D third-order tensors with the following symmetry: $\Phi_{\alpha \beta \gamma}=\Phi_{\beta \alpha \gamma}$. Moreover, $\partial \omega^{k}$ is the portion of edge on which kinematic boundary conditions apply: $\widetilde{U}^{d}$ is a given displacement and $\mathbf{H}^{d}$ is a symmetric second-order tensor related to a forced rotation on the edge. These fields are almost identical to Reissner-Mindlin kinematically compatible fields where the rotation pseudo-vector is replaced by the generalized rotation $\Phi$.

Finally, for constitutive material following local monoclinic symmetry with respect to $\left(x_{1}, x_{2}\right)$ plane (uncoupling between $\mathbf{R}$ and $(\mathbf{N}, \mathbf{M})$ ) the Bending-Gradient plate constitutive equations are written as:

$$
\left\{\begin{array}{l}
\mathrm{N}=\mathcal{A}: \mathbf{e}+\mathcal{B}: \boldsymbol{\chi} \\
\mathrm{M}={ }^{t} \mathcal{B}: \mathbf{e}+\mathcal{D}: \boldsymbol{\chi} \\
\Gamma=\mathbb{F} \therefore \mathrm{R}, \quad \text { where } \quad(\mathbb{U}-\mathbb{f} \therefore \mathbb{F}) \therefore \Gamma=0
\end{array}\right.
$$

where conventional Love-Kirchhoff stiffnesses are defined as:

$$
(\mathcal{A}, \mathcal{B}, \mathcal{D})=\left\langle\left(1, x_{3}, x_{3}^{2}\right) \boldsymbol{c}\left(x_{3}\right)\right\rangle
$$

and $\mathbb{f}$ is the generalized shear compliance tenso $\mathbb{1}$ defined as:

$$
\mathbb{f}=\int_{-\frac{h}{2}}^{\frac{h}{2}}\left(\int_{-\frac{h}{2}}^{x_{3}}\left({ }^{t} \boldsymbol{b}+z \boldsymbol{d}\right): \boldsymbol{c}(z) d z\right) \cdot \mathbf{S}\left(x_{3}\right) \cdot\left(\int_{-\frac{h}{2}}^{x_{3}} \boldsymbol{c}(z):(\boldsymbol{6}+z \boldsymbol{d}) d z\right) d x_{3}
$$

where $\mathbf{S}=\mathrm{S}_{\alpha \beta}=4 \mathcal{S}_{\alpha 3 \beta 3}$ is the out-of-plane shear compliance tensor. Since $\mathbb{f}$ is not always invertible, we introduced Moore-Penrose pseudo inverse for the shear stiffness tensor $\mathbb{F}$ :

$$
\mathbb{F}=\lim _{\kappa \rightarrow 0}(\mathbb{f} \therefore \mathbb{f}+\kappa \mathbb{l})^{-1} \therefore \mathbb{f}
$$

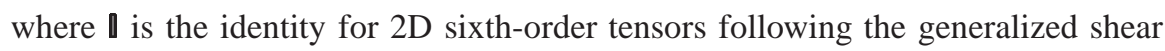
compliance $\mathbb{f}$ minor and major symmetries $\left(\square_{\alpha \beta \gamma \delta \varepsilon \zeta}=i_{\alpha \beta \varepsilon \zeta} \delta_{\gamma \delta}\right)$. The solution of the plate model must comply with the three sets of equations 16 17 18. The compliance $\mathbb{f}$ is positive. However when $\mathbb{f}$ is not definite, there is a set of solutions, up to a self-stress field.

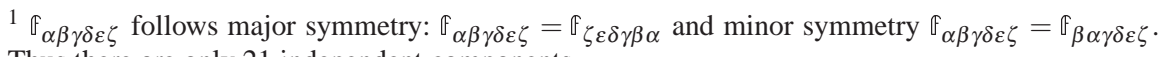
Thus there are only 21 independent components
} 
Projection of the Bending-Gradient plate model

In some cases, the Bending-Gradient is turned into a Reissner-Mindlin plate model. This is the case for homogeneous plates. Thus, we need a means to estimate the difference between both plate models. It is possible to define the exact projection of the Bending-Gradient model on a Reissner-Mindlin model.

The Reissner-Mindlin part of $\mathbb{f}$ is:

$$
\mathbb{f}^{R M}=\left(\frac{2}{3} \boldsymbol{i} \cdot \boldsymbol{i}\right) \therefore \mathbb{f} \therefore\left(\frac{2}{3} \boldsymbol{i} \cdot \boldsymbol{i}\right)
$$

$\mathbb{f}^{R M}$ can be considered as the restriction of $\mathbb{f}$ when setting warping unknowns to zero. Consequently, we introduce the pure warping part of $\mathbb{f}$ as the orthogonal complement of $\mathbb{f}^{R M}$ :

$$
\mathbb{f}^{\mathrm{W}}=\mathbb{\mathfrak { F }}-\mathbb{\mathfrak { l }}^{R M}
$$

Finally we suggest the following relative distance between the Bending-Gradient plate model and the Reissner-Mindlin one:

$$
\Delta^{R M / B G}=\frac{\left\|\mathbb{f}^{\mathrm{W}}\right\|}{\|\mathbb{f}\|}, \quad \text { where } \quad\|\mathbb{F}\|=\sqrt{\mathbb{E}_{\alpha \beta \gamma \delta \varepsilon \zeta^{\mathbb{E}} \zeta \varepsilon \delta \gamma \beta \alpha}}
$$

$\Delta^{R M / B G}$ gives an estimate of the pure warping fraction of the shear stress energy and is a criterion for assessing the need of the Bending-Gradient model. When the plate constitutive equation is restricted to a Reissner-Mindlin one, we have exactly $\Delta^{R M / B G}=0$.

\section{Application to laminates}

\subsection{Plate configuration}

We consider angle-ply laminates. Each ply is made of unidirectional fiber-reinforced material oriented at $\theta$ relative to the bending direction $x_{1}$. All plies have the same thickness and are perfectly bounded. A laminate is denoted between brackets by the successive ply-orientations along thickness. For instance $\left[0^{\circ}, 90^{\circ}\right]$ denotes a 2 -ply laminate where the lower ply fibers are oriented in the bending direction. The constitutive behavior of a ply is assumed to be transversely isotropic along the direction of the fibers and engineering constants are chosen similar to those of [1]:

$E_{L}=25 \times 10^{6} \mathrm{psi}, \quad E_{T}=1 \times 10^{6} \mathrm{psi}, \quad G_{L T}=0.5 \times 10^{6} \mathrm{psi}, \quad G_{T T}=0.4 \times 10^{6} \mathrm{psi}$,

$$
v_{L T}=v_{T T}=0.25
$$


where $G_{T T}$ has been changed to preserve transversely isotropic symmetry. $L$ is the longitudinal direction oriented in the $\left(x_{1}, x_{2}\right)$ plane at $\theta$ with respect to $\widetilde{\boldsymbol{e}}_{1}, T$ is the transverse direction.

Distance between the Reissner-Mindlin and the Bending-Gradient model

In Table 1 are given the values of $\Delta^{R M / B G}$ for the laminates considered in this work. For a single ply, the criterion is zero since the Bending-Gradient model is exactly a Reissner-Mindlin model in this case. However, when there are several plies, the distance is greater than $10 \%$. Thus with these laminates, the shear constitutive equation cannot be reduced to a Reissner-Mindlin behavior.

$$
\begin{array}{c|cccc}
\text { Stack } & {\left[0^{\circ}\right]\left[0^{\circ}, 90^{\circ}\right]} & {\left[30^{\circ},-30^{\circ}\right]_{s}} & {\left[30^{\circ},-30^{\circ}\right]} \\
\hline \Delta^{R M / B G} & 0 & 16.0 \% & 16.0 \% & 23.9 \%
\end{array}
$$

Table 1 The criterion $\Delta^{R M / B G}$ for several laminates

\subsection{Cylindrical bending}

Pagano [1] gives an exact solution for cylindrical bending of simply supported composite laminates. We choose the same configuration for the Bending-Gradient model. The plate is invariant and infinite in $x_{2}$ direction. It is out-of-plane loaded with $q_{3}\left(x_{1}\right)=-q_{0} \sin \kappa x_{1}$ where $\lambda=1 / \kappa$ is the wavelength of the loading (Figure 2, The plate is simply supported at $x_{1}=0$ and $x_{1}=L$ with traction free edges:

$$
U_{3}(0)=0, U_{3}(L)=0, \mathbf{M}(0)=0, \mathbf{M}(L)=0, \mathbf{N}(0) \cdot \boldsymbol{e}_{1}=\mathbf{0}, \mathbf{N}(L) \boldsymbol{e}_{1}=\mathbf{0}
$$

$\mathrm{M}_{22}(0)=\mathrm{M}_{22}(L)=0$ is the additional boundary condition compared to the ReissnerMindlin plate model. This additional boundary condition takes into account free edge effects similar to those described in 17 f for periodically layered laminate. The resolution is provided in details in [18].

Closed-form solutions using the Reissner-Mindlin model were also derived in order to compare them with the Bending-Gradient. The work of Whitney [19] was used for deriving transverse shear stress distributions and shear correction factors were taken into account into the shear constitutive equation of the Reissner-Mindlin plate model.

A comparison with a finite elements solution was also performed on ABAQUS [20]. Since the Bending-Gradient is an Equivalent Single Layer theory, conventional shell elements were chosen (3 displacements and 3 rotations). Transverse shear fields with shell elements in ABAOUS are derived using an approach very similar 


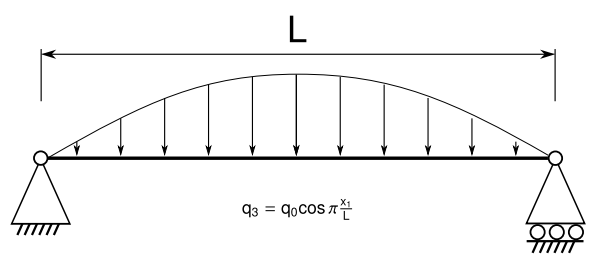

Fig. 2 Pagand's cylindrical bending configuration

to [19] where it is furthermore assumed that the plate overall constitutive equation is orthotropic with respect to the main bending direction. $S 4$, linear quadrangle with full integration elements, were used. A convergence test was performed. This study enforced the typical size of an element $l_{\text {char }}=h / 5$ where $h$ is the plate thickness. For instance when the slenderness is $h / L=1 / 4$ there are 20 elements. Figure 3 shows a typical deformation of this mesh. Periodicity was enforced on lateral edges of the strip in Figure 3 by equating corresponding rotations and displacements. Finally, section integration is performed during the analysis.

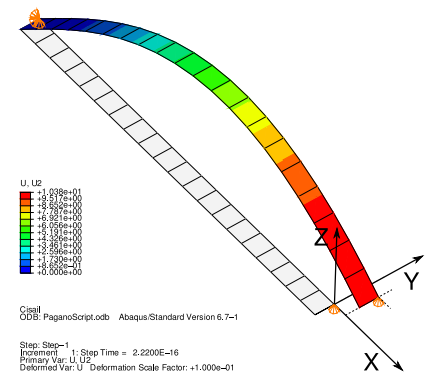

Fig. 3 Finite Element undeformed and deformed mesh for an anisotropic laminate

Two error estimates are introduced: the first one for the transverse shear part of the stresses for which we introduce the following seminorm:

$$
\|\boldsymbol{\sigma}\|^{2}=\int_{-\frac{L}{2}}^{\frac{L}{2}} \int_{-\frac{h}{2}}^{\frac{h}{2}} \sigma_{\alpha 3} S_{\alpha 3 \beta 3} \sigma_{\beta 3} d x_{3} d x_{1}
$$

and we define the relative error as: $\Delta(\boldsymbol{\sigma})=\frac{\left\|\boldsymbol{\sigma}^{E x}-\boldsymbol{\sigma}\right\|}{\left\|\boldsymbol{\sigma}^{E x}\right\|}$, where $\boldsymbol{\sigma}^{E x}$ is the exact shear stress distribution from Pagano [1, 21, 22]. The second one is the mid-span deflection relative error: $\Delta\left(U_{3}\right)=\frac{U_{3}^{E x}(L / 2)-U_{3}(L / 2)}{U_{3}^{E x}(L / 2)}$, where $U_{3}^{E x}\left(x_{1}\right)$ is the plate deflection taken for the exact solution. 


\subsection{Results}

First, we consider a skew-symmetric cross ply $\left[0^{\circ}, 90^{\circ}\right]$ laminate. In this case, the plate configuration fulfills the assumptions made for the finite elements approximation (orthotropic laminate). In Figure 4 shear stress distribution in both directions are plotted for the exact solution from Pagano [1] $\boldsymbol{\sigma}^{E x}$, the Bending-Gradient solution $\boldsymbol{\sigma}^{(\mathrm{R})}$, Whitnev's shear distribution $\boldsymbol{\sigma}^{(Q), W}$ and the finite elements solution $\boldsymbol{\sigma}^{(Q), F E}$. The slenderness ratio is set to $L / h=4$ as conventionally done when benchmarking plate models. The three approximate solutions yield the same distribution. The discrepancy with the exact solution is well-known and associated to edge effects. In Figure 5 the transverse shear stress distribution error $\Delta(\boldsymbol{\sigma})$ versus the slen-

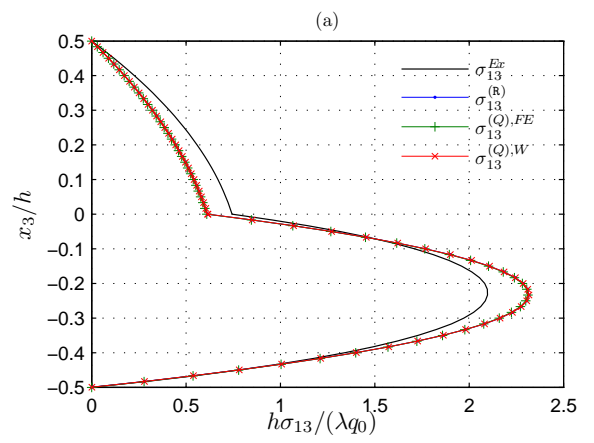

Fig. 4 Normalized shear distribution $\sigma_{13}$ at $x_{1}=0$ for a $\left[0,90^{\circ}\right]$ laminate, $L / h=4$

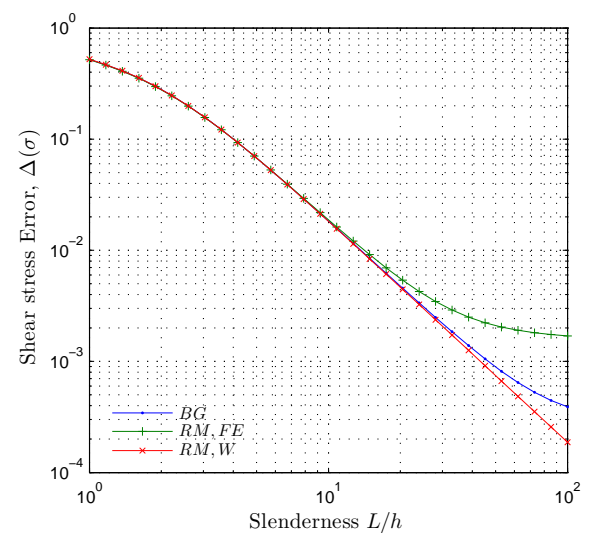

Fig. 5 Shear stress distribution error versus slenderness ratio for a $\left[0^{\circ}, 90^{\circ}\right]$ laminate

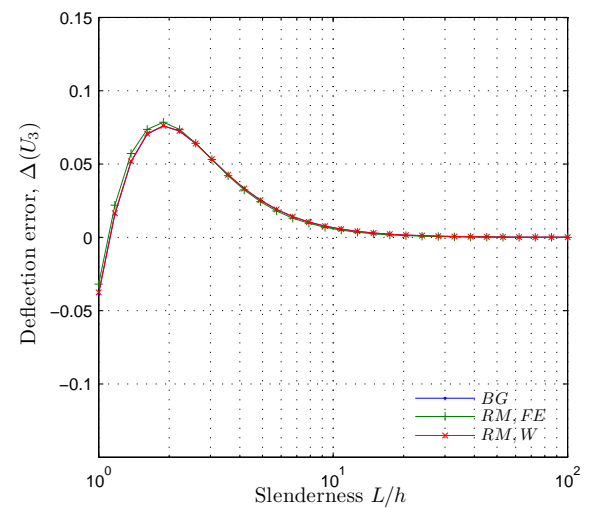

Fig. 6 Deflection error versus slenderness ratio for a $\left[0,90^{\circ}\right]$ laminate 
derness ratio $L / h$ is plotted for the Bending-Gradient solution $(B G)$, the finite elements solution $(R M, F E)$ and the closed-form Reissner-Mindlin solution $(R M, W E)$. In this case, Whitnev's solution converges with $L / h$ whereas finite elements and Bending-Gradient approximations do not converge and lead to rather small errors $\left(\simeq 10^{-3}\right)$. In Figure 6 the mid-span deflection error is also plotted versus the slenderness ratio. The three approximate solutions yields almost the same error.

We consider now a symmetric and non-orthotropic $\left[30^{\circ},-30^{\circ}\right]_{s}$ laminate. This configuration does not comply with the assumptions made for the finite elements approach. In Figure 7 shear distributions are compared to the exact solution. The Bending-Gradient solution remains close to the exact solution. However finite elements and Whitnev's solution yield different distributions which are not as accurate as the Bending-Gradient. More precisely, in Direction 2, the FE solution does not capture the change of slope associated to the change of ply orientation. In Direction 1 the macroscopic equilibrium is respected for all approximated solutions $\left(\left\langle\sigma_{13}\right\rangle=Q_{1}\right)$. However in Direction 2 we can see that $\left\langle\sigma_{23}^{F E}\right\rangle \neq Q_{2}$ for both finite elements and Whitney's solution. In Figure 8 the transverse shear stress distribution
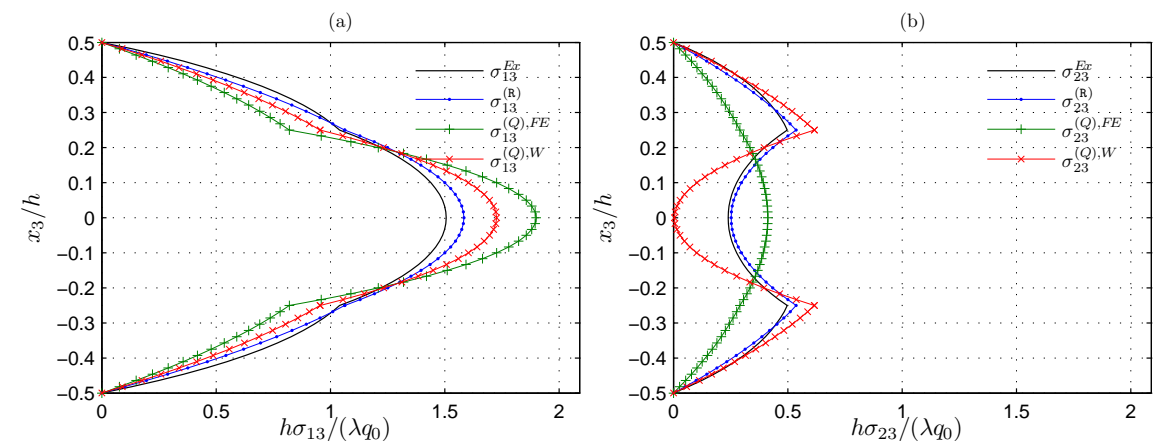

Fig. 7 Normalized shear distribution in both directions at $x_{1}=0$ for a $\left[30^{\circ},-30^{\circ}\right]_{s}$ laminate, $L / h=$ 4, a) $\sigma_{13}$ b) $\sigma_{23}$.

error versus the slenderness ratio is plotted. Contrary to the finite elements solution and Whitnev's solution, the Bending-Gradient solution converges when the plate is slender. More precisely we have: $\Delta\left(\boldsymbol{\sigma}^{B G}\right) \propto\left(\frac{h}{L}\right)^{2}$ in this case. In Figure 9 the mid-span deflection error is also plotted versus the slenderness ratio. The BendingGradient solution is the most accurate one for conventional slenderness.

Finally, in Figure 10 the comparison is made for a non-symmetric and nonorthotropic ply $\left[30^{\circ},-30^{\circ}\right]$. Again, this configuration does not comply with the assumptions made for the finite elements approach. The Bending-Gradient solution remains close to the exact solution and Whitney's solution yields acceptable results (except a mismatch for $\sigma_{23}^{(\boldsymbol{Q}), W}$ ). However in this case, finite elements yields inappropriate results: in Direction 1 the stress distribution does not respect macroscopic equilibrium $\left\langle\sigma_{13}^{F E}\right\rangle \neq Q_{1}$. We checked nevertheless that FE nodal forces ful- 


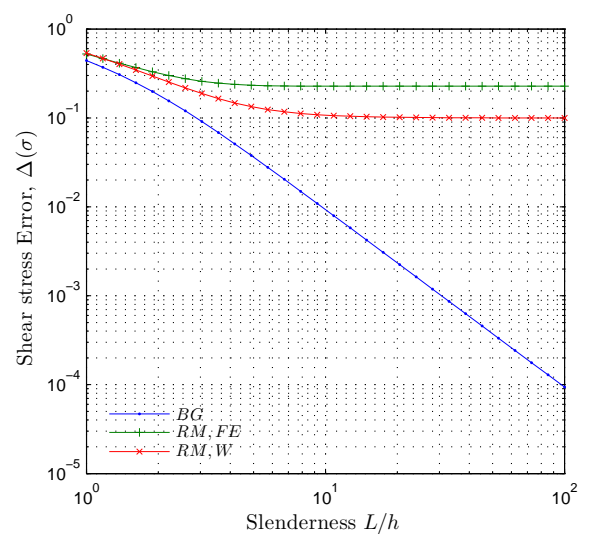

Fig. 8 Shear stress distribution error versus slenderness ratio for a $\left[30^{\circ},-30^{\circ}\right]_{s}$ laminate

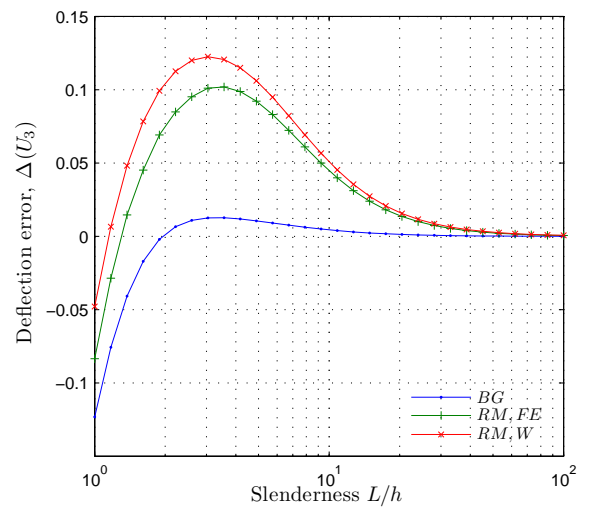

Fig. 9 Deflection error versus slenderness ratio for a $\left[30^{\circ},-30^{\circ}\right]_{S}$ laminate

fills macroscopic equilibrium. This inaccuracy is also clear in Figure 5 showing
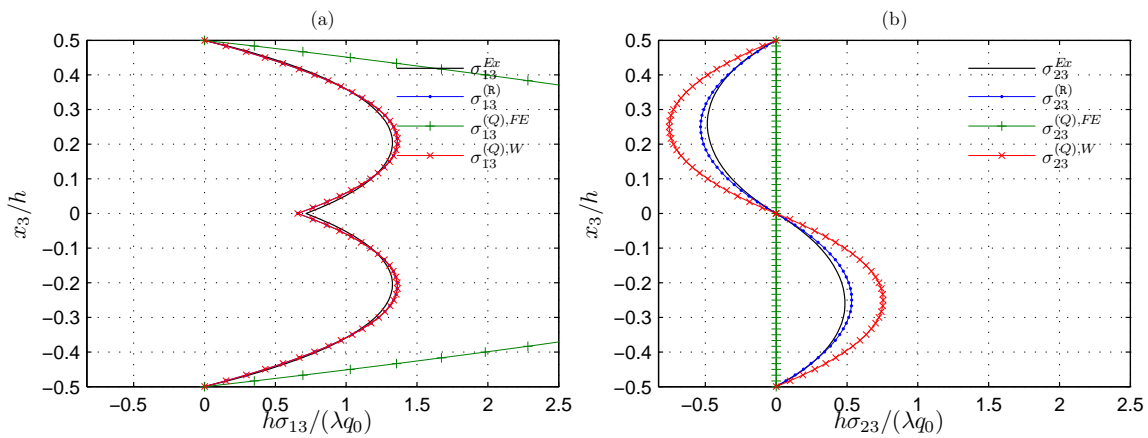

Fig. 10 Normalized shear distribution in both directions at $x_{1}=0$ for a $\left[-30^{\circ}, 30^{\circ}\right]$ laminate, $L / h=4$, a) $\sigma_{13}$ b) $\sigma_{23}$.

the transverse shear stress distribution error versus the slenderness ratio whereas the Bending-Gradient converges as $\Delta\left(\boldsymbol{\sigma}^{B G}\right) \propto\left(\frac{h}{L}\right)^{2}$ and both the Whitney and finite elements solutions lead to non-negligible errors. Again, in Figure 12 the deflection error indicates that FE are too compliant and that the Bending-Gradient is more accurate than the Reissner-Mindlin solution. 


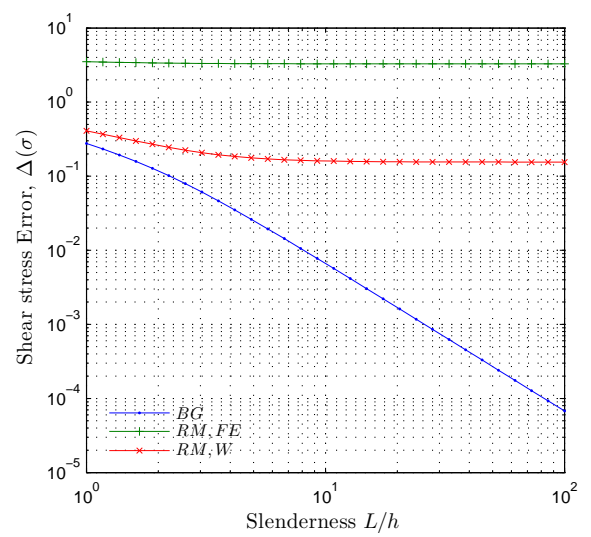

Fig. 11 Shear stress distribution error versus slenderness ratio for a $\left[30^{\circ},-30^{\circ}\right]$ laminate

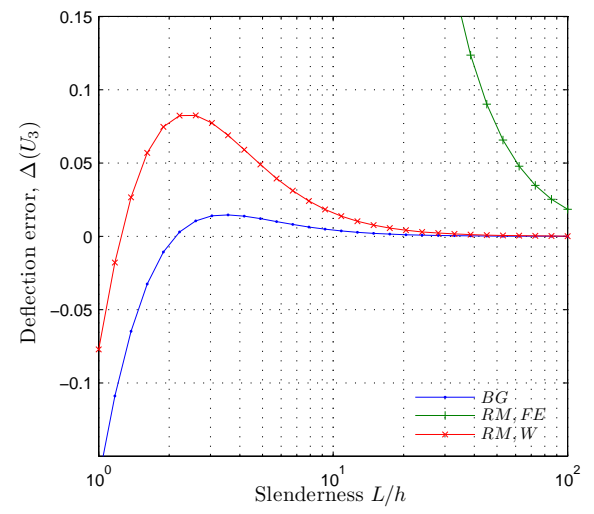

Fig. 12 Deflection error versus slenderness ratio for a $\left[30^{\circ},-30^{\circ}\right]$ laminate

\subsection{Discussion}

We have compared three approaches for deriving an approximation of the exact solution for cylindrical bending suggested by Pagano [1, 21, 22].

First we derived closed-form solutions for the Reissner-Mindlin model using shear correction factors and shear distributions from [19]. This approach yields a fair estimation of the deflection and shear distributions in cylindrical bending but it is not as accurate as the Bending-Gradient approximation in most cases. The main limitation of this approach is the cylindrical bending assumption. It is not sure that shear correction factors and shear distributions will remain valid with more general plate boundary conditions, especially involving torsion, whereas the BendingGradient theory is not limited to cylindrical bending.

Second, we implemented a finite elements approximation, using conventional shell elements. This approach assumes both cylindrical bending and orthotropy in the same direction. When these assumptions are not valid, the results might be really affected both for deflection and stress distribution as demonstrated for the $\left[30^{\circ},-30^{\circ}\right]$ laminate.

Finally, the Bending-Gradient solution was presented. This approach enables the derivation of stress distributions and gives good enough deflection and stress distribution estimates whatever the plate configuration and the bending direction are. Moreover, it was numerically demonstrated that in some configurations the Bending-Gradient solution converges with the slenderness ratio.

Let us state this convergence condition precisely. We chose to neglect the gradient of membrane stress $\mathbf{N} \otimes \boldsymbol{\nabla}$ since it is not related to macroscopic stress. In the cylindrical bending configuration, the membrane stress is reduced to $\mathrm{N}_{22}$. When $\mathrm{N}_{22}=0$, the membrane stress gradient vanishes. This is the case for $\left[30^{\circ},-30^{\circ}\right]_{s}$ and $\left[30^{\circ},-30^{\circ}\right]$ since they are balanced laminates (as many $\theta$ plies as $-\theta$ plies). 
In these cases, the Bending-Gradient solution converges because the stress fields related to the membrane stress gradient do not contribute to the final solution. It is possible to generalize this result to any boundary conditions with mirror symmetric laminates $(\boldsymbol{B}=0)$ for which the membrane problem is fully uncoupled from the out-of-plane problem. With these laminates, the Bending-Gradient solution is the Saint-Venant solution for an out-of-plane loaded plate.

\section{Conclusion}

In the present paper, we derived a new plate theory, the Bending-Gradient theory, which is the extension of Reissner-Mindlin theory to laminated plates. Comparison with the exact solution for cylindrical bending of cross ply laminates demonstrates that the Bending-Gradient gives good predictions of both deflection and shear stress distributions in any material configuration. It is also the Saint-Venant solution when membrane stresses are fully uncoupled from bending moments and generalized shear stresses. Finally, with usual laminated plates, we pointed out that the Bending-Gradient cannot be reduced to a Reissner-Mindlin plate model.

Several outlooks are under consideration. First, this plate theory can be extended to periodic plates such as sandwich panels [23, 24]. Second, the estimation of the influence of the membrane stress gradient on the quality of the shear stress estimation should be studied in detail.

\section{References}

[1] N. Pagano, Exact Solutions for Composite Laminates in Cylindrical Bending, Journal of Composite Materials 3 (3) (1969) 398-411.

[2] D. Caillerie, Thin elastic and periodic plates., Mathematical Methods in the Applied Sciences 6 (2) (1984) 159 - 191, ISSN 01704214.

[3] T. Lewinski, Effective Models Of Composite Periodic Plates .1. Asymptotic Solution, International Journal Of Solids And Structures 27 (9) (1991) 11551172.

[4] J. N. Reddy, On Refined Computational Models Of Composite Laminates, International Journal For Numerical Methods In Engineering 27 (2) (1989) 361382.

[5] E. Carrera, Theories and finite elements for multilayered, anisotropic, composite plates and shells, Archives Of Computational Methods In Engineering 9 (2) (2002) 87-140.

[6] A. Diaz Diaz, J.-F. Caron, R. P. Carreira, Model for laminates, Comptes Rendus de l'Académie des Sciences - Series IIB - Mechanics 329 (12) (2001) 873-879, ISSN 1620-7742. 
[7] J. F. Caron, A. D. Diaz, R. P. Carreira, A. Chabot, A. Ehrlacher, Multi-particle modelling for the prediction of delamination in multi-layered materials, Composites Science And Technology 66 (6) (2006) 755-765.

[8] A. Diaz Diaz, J. F. Caron, A. Ehrlacher, Analytical determination of the modes I, II and III energy release rates in a delaminated laminate and validation of a delamination criterion, Composite Structures 78 (3) (2007) 424-432.

[9] T.-K. Nguyen, K. Sab, G. Bonnet, First-order shear deformation plate models for functionally graded materials, Composite Structures 83 (1) (2008) 25-36.

[10] T.-K. Nguyen, K. Sab, G. Bonnet, Green's operator for a periodic medium with traction-free boundary conditions and computation of the effective properties of thin plates, International Journal of Solids and Structures 45 (25-26) (2008) 6518-6534, ISSN 0020-7683.

[11] E. Reissner, The effect of transverse shear deformation on the bending of elastic plates, Journal of Applied Mechanics 12 (1945) 68-77.

[12] J.-F. Caron, K. Sab, Un nouveau modèle de plaque multicouche épaisse. A new model for thick laminates, Comptes Rendus de l'Académie des Sciences - Series IIB - Mechanics 329 (8) (2001) 595-600, ISSN 1620-7742.

[13] V.-T. Nguyen, J.-F. Caron, K. Sab, A model for thick laminates and sandwich plates, Composites Science and Technology 65 (3-4) (2005) 475 - 489, ISSN 0266-3538.

[14] C. Boutin, Microstructural effects in elastic composites, International Journal Of Solids And Structures 33 (7) (1996) 1023-1051.

[15] N. Buannic, P. Cartraud, Higher-order effective modeling of periodic heterogeneous beams. I. Asymptotic expansion method, International Journal of Solids and Structures 38 (40-41) (2001) 7139-7161, ISSN 0020-7683.

[16] A. Lebée, K. Sab, A bending gradient model for thick plates, Part I: Theory, submitted.

[17] A. Lebée, K. Sab, A Cosserat multiparticle model for periodically layered materials, Mechanics Research Communications 37 (3) (2010) 293-297, ISSN 0093-6413.

[18] A. Lebée, K. Sab, A bending gradient model for thick plates, Part II: Closedform solutions for cylindrical bending, submitted .

[19] J. Whitney, Stress Analysis of Thick Laminated Composite and Sandwich Plates, Journal of Composite Materials 6 (4) (1972) 426-440.

[20] ABAQUS, ABAQUS/Standard user's manual, version 6.7, 2007.

[21] N. Pagano, Exact Solutions for Rectangular Bidirectional Composites and Sandwich Plates, Journal of Composite Materials 4 (1) (1970) 20-34.

[22] N. Pagano, Influence of Shear Coupling in Cylindrical. Bending of Anisotropic Laminates, Journal of Composite Materials 4 (3) (1970) 330-343.

[23] A. Lebée, K. Sab, Reissner-Mindlin Shear Moduli of a Sandwich Panel with Periodic Core Material, in: Mechanics of Generalized Continua, vol. 21 of Advances in Mechanics and Mathematics, Springer New York, 169-177, 2010.

[24] A. Lebée, K. Sab, Transverse shear stiffness of a chevron folded core used in sandwich construction, International Journal of Solids and Structures 47 (1819) (2010) 2620-2629, ISSN 0020-7683. 